\title{
The importance of residual complexity
}

\begin{tabular}{|c|c|}
\hline $\begin{array}{l}\text { lead } \\
\text { compounds } \\
\text { should be } \\
\text { required } \\
\text { to undergo } \\
\text { advanced } \\
\text { purity analysis } \\
\text { at early stages }\end{array}$ & $\begin{array}{l}\text { Residual complexity is defined as } \\
\text { the inherent deviation from absolute } \\
\text { purity of purported single chemical } \\
\text { entities of metabolomic and synthetic } \\
\text { origin that require chromatographic } \\
\text { isolation. This phenomenon can } \\
\text { have serious implications in drug } \\
\text { discovery, and an example of residual } \\
\text { complexity presented in a new paper } \\
\text { in the Journal of Organic Chemistry } \\
\text { highlights the inadequacy of the } \\
\text { current gold standard for assessing } \\
\text { analytical purity - high-performance } \\
\text { liquid chromatography with ultra- } \\
\text { violet-visible spectroscopic detection }\end{array}$ \\
\hline
\end{tabular}

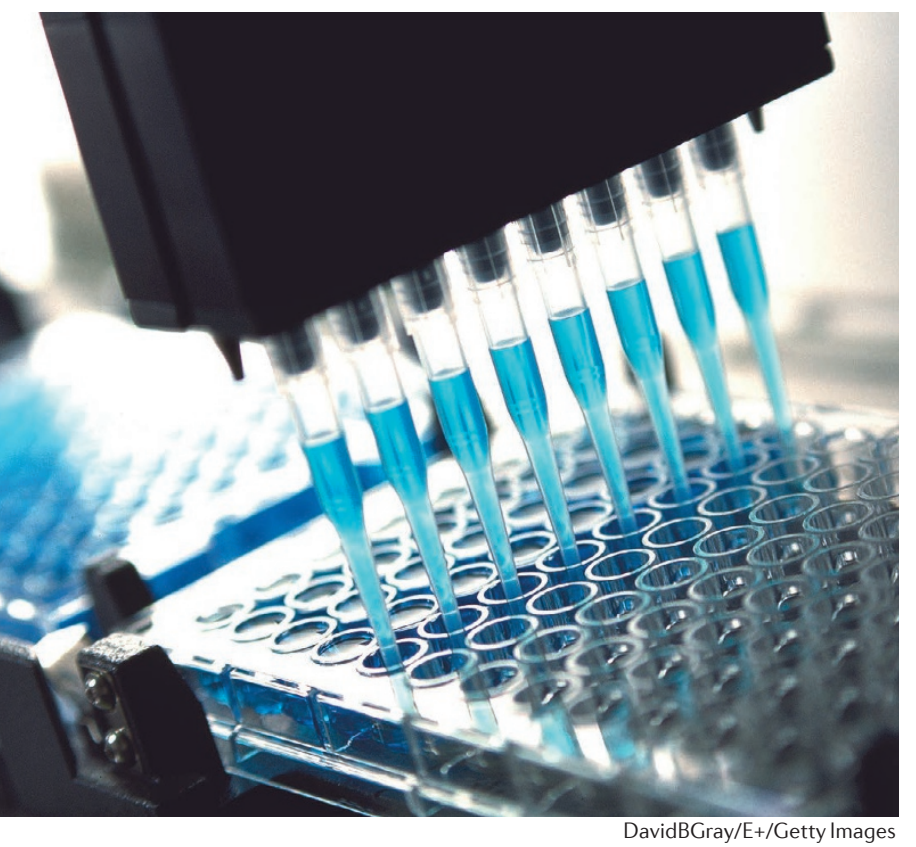

(HPLC UV-VIS) - and indicates that quantitative NMR (qNMR) spectroscopy could be more useful.

Unidentified impurities in the sample of a compound used for screening, even when in low abundance, can exert a major effect on biological activity. Both misidentification (where activity is real, but structural identity is wrong) and misassignment (where activity is assigned to the wrong component of a mixture) are underappreciated in drug discovery research. In their latest study, Choules et al. describe an example of residual complexity that led to the misassignment of activity to an inactive component that initially appeared to be highly pure.

The researchers isolated a new diketopiperazine, rufomyazine, from an actinomycete strain and found it exhibited a minimum inhibitory concentration (MIC) of approximately $5 \mu \mathrm{M}$ against Mycobacterium tuberculosis. Although not as potent as the previously isolated antibiotic rufomycin (which has a MIC of $\sim 0.02 \mu \mathrm{M}$ ), the smaller molecular size of rufomyazine and likelihood of a simpler structure-activity relationship led the researchers to investigate rufomyazine further as a potential lead compound. They synthesized the molecule but determined that the synthetic product, although spectrally identical to the natural product, was inactive.
Extensive analytical studies using techniques including qNMR and liquid chromatography-mass spectrometry (LC-MS) showed that the unexplained activity of the isolated material was the result of very minor contamination - only $0.24 \%$ by weight - by an analogue of the highly active compound rufomycin. So, an impurity percentage much lower than the natural abundance of ${ }^{13} \mathrm{C}(1.1 \%)$ had a major impact on the observed activity of a sample in this case. Furthermore, the percentage impurity is more than 20 -fold lower than the $95 \%$ purity level that is often used by journals as a minimum threshold of purity.

While the issue of residual complexity is particularly relevant for natural product drug discovery, it is also important to keep in mind in synthetic medicinal chemistry. The authors conclude that, in order to prevent futile downstream development efforts, lead compounds should be required to undergo advanced purity analysis at early stages using qNMR and LC-MS, which are more specific and sensitive, respectively, than the current gold standard, HPLC UV-VIS.

Rebecca Kelsey

ORIGINAL ARTICLE Choules, M. P. et al. Residual complexity does impact organic chemistry and drug discovery: the case of rufomyazine and rufomycin.J. Org. Chem. https://doi.org/10.1021/ acs.joc. 8 b00988 (2018) 